\title{
PRÁTICA DO PSICÓLOGO EM INTERVENÇÃO PRECOCE NA SAÚDE MATERNO-INFANTIL ${ }^{1}$
}

\author{
Ana Cristina Barros da Cunha* \\ Julita Benevides ${ }^{\#}$
}

\begin{abstract}
RESUMO. Tomando como referência a abordagem bioecológica do desenvolvimento proposto por U. Bronfenbrenner e os pressupostos da Política Nacional de Humanização do SUS, objetivou-se investigar as práticas de intervenção precoce em maternidades públicas de referência na cidade do Rio de Janeiro e a atuação de psicólogos nesse tipo de prática no campo da Saúde Materno-infantil. Foram entrevistados dez psicólogos de seis maternidades para gestação de alto risco e feita observação das maternidades usando um protocolo de observação e um roteiro de entrevista elaborados pelas autoras. Os dados revelaram que existem diferentes práticas de intervenção precoce nas instituições visitadas e que os psicólogos compreendiam intervenção precoce como um trabalho focado no vínculo mãe-bebê, reconhecendo-a como prática técnico-profissional que facilitaria a promoção da saúde materno-infantil.
\end{abstract}

Palavras-chave: Intervenção precoce; saúde materno-infantil; atuação do psicólogo.

\section{PSYCHOLOGIST PRACTICE IN EARLY INTERVENTION IN THE MATERNAL AND CHILD HEALTH}

\begin{abstract}
With reference to the bio-ecological approach of humam development proposed by U. Bronfenbrenner and the assumptions of the National Humanization Policy of SUS, this article aimed to investigate the practices of early intervention in public hospitals in the Rio de Janeiro city and the role of psychologists in this type of the practice in the Maternal and Child Health area. We interviewed 10 psychologists in six high-risk pregnancy hospitals and we observed these hospitals using an observation protocol and an interview guide developed by the authors. The data revealed that there are different practices of early intervention in the institutions visited and the psychologists understood the early intervention as a work focused on maternal and child bonding, recognizing it is as a technical and professional practice that would facilitate the promotion of maternal and child health.
\end{abstract}

Key words: Early Intervention; maternal and child health; psychologist performance.

\section{PSICÓLOGO EN LA INTERVENCIÓN PRECOZ EN SALUD MATERNO-INFANTIL}

RESUMEN. Defendemos la investigación de intervención en la interdisciplinaridad relacionando la psicología, el conocimiento y el desarrollo de Tomando como referencia el enfoque bio-ecológica del desarrollo humano propuesto por U. Brofenbrenner y los supuestos de la Policía Nacional de Humanización del SUS, el objetivo fue investigar las prácticas de intervención precoz en los hospitales de maternidad de referencia en la ciudad de Rio de Janeiro y la práctica de los psicólogos en este tipo de práctica en el campo de la salud materno-infantil. Entrevistamos a 10 psicólogos de seis hospitales de embarazo de alto riesgo y observamos los hospitales utilizando un protocolo de observación y una guía de entrevista, desarrollados por los autores. Los datos revelaron que existen diferentes prácticas para la intervención precoz en las instituciones visitadas y los psicólogos entendían esa intervención como una práctica centrada en el vínculo madre-hijo, reconociendo como una práctica técnico-profesional que faciliten la promoción de la salud materno-infantil.

Palabras-clave: Intervención precoz; salud materna e infantil; actuación del psicólogo.

A prática clínica no campo do desenvolvimento infantil precoce é um trabalho de intervenção delicado e complexo, já que implica na adoção de medidas de urgência ante a fragilidade da vida do bebê e, ao

\footnotetext{
Apoio: CNPq e FAPERJ.

Doutora em Psicologia Social e do Desenvolvimento pela Universidade Federal do Espírito Sando (2004); docente do Departamento de Psicologia Clínica no Instituto de Psicologia da Universidade Federal do Rio de Janeiro UFRJ, Brasil.

\# Psicóloga pela Universidade Federal do Rio de Janeiro, Brasil.
} 
mesmo tempo, a necessidade de aguardar o momento adequado para avaliar corretamente os resultados das intervenções realizadas. Nesse trabalho é imprescindível o envolvimento da díade mãe-bebê, que, em sua relação simbiótica, por vezes demanda intervenções clínicas que consigam equacionar o tempo exato de agir com as necessidades de saúde do par.

Segundo Franco (2007), a importância cada vez maior dada ao período mais precoce do desenvolvimento de uma criança tem levado ao questionamento acerca dos cuidados dispensados ao bebê e à procura da máxima eficácia na resposta às suas necessidades. Nessa perspectiva, o conceito de intervenção precoce surge como uma possibilidade intencional de intervir junto ao desenvolvimento infantil, de maneira a garantir que este processo ocorra da melhor maneira possível, especialmente para aquelas crianças que, por alguma perturbação existente, ou apenas potencial, se encontram em risco de ter o seu desenvolvimento afetado.

Dessa forma se busca, com a prática da intervenção precoce, minimizar fatores de risco que podem resultar em déficits ou problemas de desenvolvimento, de modo a possibilitar à criança desenvolver-se em todo o seu potencial e constituir-se enquanto sujeito psíquico. Neste sentido, diferentes autores concordam que as intervenções precoces produzem resultados superiores quando comparados aos efeitos de intervenções tardias (Hallal, Marques \& Braccialli, 2008; Lampreia, 2007). Tal hipótese se justifica pela ideia de que a neuroplasticidade cerebral no período mais precoce do desenvolvimento infantil é maior, possibilitando, à criança alcançar ganhos mais significativos, consistentes e duradouros em termos de aquisição de habilidades e competências futuras.

Este fato é de grande importância quando considerado pela perspectiva teórico-metodológica da abordagem ecológica do desenvolvimento proposta por Bronfenbrenner (1989). Segundo este autor, o desenvolvimento humano se dá de maneira contextual, ou seja, é um processo dinâmico que ocorre sob influência dos diferentes contextos ecológicoambientais em que o individuo, direta ou indiretamente, está interagindo. Nessa perspectiva, o desenvolvimento é compreendido como um conjunto de processos em que as particularidades da pessoa e do ambiente interagem, produzindo constâncias e mudanças nas características desta pessoa no curso de sua vida (Bronfenbrenner, 1989).

De acordo com a teoria bioecológica do desenvolvimento, Graminha e Martins (1997) também consideram as multideterminações ambientais em sua intima relação com os processos psicológicos e com os fatores biológicos, que juntos irão conduzir o processo de desenvolvimento humano. Os autores destacam fatores tais como a genética, a nutrição, a atitude dos adultos e oportunidades diferentes de experiências sociais, que determinam o desenvolvimento infantil e possíveis riscos a este processo.

Além dos contextos ecológicos ou ambientais em que o desenvolvimento ocorre, as relações interpessoais também desempenham papel fundamental na promoção do desenvolvimento. Bronfenbrenner (1979/1996) destaca a importância das relações entre as pessoas e da formação de díades a partir da presença de uma relação interpessoal recíproca, considerando-as elementos-chave para o desenvolvimento humano. Segundo Martins e Szymanski (2004), a díade primária consiste naquela que, mesmo quando os dois membros não estão próximos, ainda continua existindo fenomenologicamente. Como exemplo, pode-se destacar a díade mãe-bebê, caso em que, mesmo estando separados os seus componentes, um destes (mãe ou bebê) influencia o comportamento do outro.

Assim, sob a ótica da teoria bioecológica, uma intervenção no contexto primário da vida de um bebê tem o poder de influenciar diretamente o percurso de seu desenvolvimento, seja esta intervenção sobre o ambiente, sobre a mãe, sobre o próprio bebê ou, ainda, sobre a relação mãe-bebê, de maneira mais ampla. Pode-se considerar esse tipo de intervenção como uma intervenção precoce, não apenas pelo momento em que ocorre, mas, sobretudo, pelos efeitos que produzirá no desenvolvimento infantil, visto que as mudanças e modificações adotadas podem repercutir na prevenção aos fatores de risco e na promoção de mecanismos de proteção ao desenvolvimento.

A discussão acerca do conceito e prática da intervenção precoce é mais atual no Brasil, já que, historicamente, a intervenção sobre o desenvolvimento precoce já se constituiu em uma prática compensatória e higienista. Datam da década de 1960 os primeiros programas de intervenção precoce, os quais, baseados na teoria construtivista e interacionista de Piaget, conferiam um papel preponderante ao contexto em que a criança se desenvolvia. Estudos sobre os "períodos sensíveis do desenvolvimento" (Hunt, 1961; Bloom, 1964 apud Tegethof, 2007), conceito que já indicava desde então o que hoje se entende por neuroplasticidade dos primeiros anos de vida, contribuíram fortemente para a divulgação da obra de Piaget no que se refere ao caráter precoce das intervenções. Neste contexto, contudo, estes programas eram chamados programas de educação 
compensatória, e destinavam-se a atender crianças em desvantagem socioeconômica, acreditando-se que os problemas de desenvolvimento decorrentes da pouca estimulação do ambiente, característicos do meio social de origem, poderiam ser compensados (Tegethof, 2007). Já os programas brasileiros tornaram-se mais expressivos a partir das décadas de 1970 e 1980, quando eram vinculados aos programas de instituições de educação especial, como os institutos para cegos e/ou para surdos, além das associações de familiares, como a APAE.

Observando a construção do conceito e da prática de intervenção precoce na perspectiva histórica, podemos compreender melhor como essas práticas são empregadas hoje em dia no contexto da saúde no Brasil. Analisando a atual Política Nacional de Humanização do Sistema Único de Saúde Brasileiro (SUS), também chamada HumanizaSUS (Brasil, 2004b), verifica-se que ela constitui um novo modelo de assistência em saúde pública, o qual tende a priorizar estratégias de promoção e de prevenção em saúde, além da melhoria dos serviços de reabilitação. Especificamente, na área da saúde materno-infantil encontram-se cada vez mais programas e políticas integradas ao SUS que, embora não tragam cunhadas em si o termo específico de "intervenções precoces", orientam-se pela perspectiva da atenção integral à saúde da criança, elencando uma série de estratégias que visam garantir condições de desenvolvimento saudável para mães e bebês no âmbito da saúde pública.

Como parte integrante dessa política destaca-se um importante documento do Ministério da Saúde intitulado "Agenda de Compromissos para a Saúde Integral da Criança e Redução da Mortalidade Infantil”, lançado em 2004, que inclui um tópico relativo à atenção à criança portadora de deficiência, que sugere a inclusão da intervenção precoce como prática preventiva (Brasil, 2004a). Nesse tópico está definida claramente a importância da atenção integral e multiprofissional à criança com deficiência, com vistas a favorecer a detecção precoce dos problemas de desenvolvimento em tempo oportuno que possibilite a adoção de ações eficazes de diagnóstico e intervenção precoce, de habilitação e reabilitação e de promoção de saúde e prevenção de agravos para minimizar as consequências da deficiência.

Outro tópico importante que também é abordado por aquele documento e atravessa a questão da intervenção precoce é o que diz respeito aos fatores de risco. Esse documento ressalta a importância de que tanto as unidades de saúde quanto as equipes de Saúde da Família e de Atenção Básica estejam capacitadas a identificar situações de risco desde o período prénatal, principalmente através do cuidado com o recémnascido, de maneira a priorizar o atendimento às situações mais graves e às crianças mais vulneráveis (Brasil, 2004b).

Observamos assim que a existência de fatores de risco constitui-se como um primeiro alerta aos profissionais de saúde materno-infantil sobre a necessidade de uma intervenção precoce, visto que se configuram como situações preditivas de ameaças ao desenvolvimento infantil e à saúde física e mental da mãe e do bebê. A partir daí seriam estabelecidas estratégias de cuidado e programas de acompanhamento adequados, com vista à evolução de cada caso, com a indicação de uma intervenção precoce ou não. Considerando que profissionais da área médica têm a possibilidade de identificar crianças expostas a fatores de risco, Graminha e Martins (1997) destacam a importância de que estes profissionais sejam conscientizados da necessidade de encaminhamento a serviços que possibilitem o monitoramento do desenvolvimento destas crianças.

Entre as linhas de cuidado preconizadas pelo SUS destacam-se as que representam ações significativas no âmbito das intervenções precoces, as quais, neste estudo, serão analisadas com o objetivo de discutir como essas ações se relacionam com os princípios fundamentais de humanização do SUS e se articulam com o tema da intervenção precoce. Entre as principais linhas de cuidado relacionadas com o campo da intervenção precoce destacam-se sete, as quais são apresentadas e descritas a seguir:

1) Atenção humanizada e qualificada à gestante e ao recém-nascido, que consiste em oferecer determinado nível de qualidade de que geralmente o ser humano necessitaria para ser bem atendido e cuidado em suas necessidades. Nesse contexto, contempla-se não apenas os problemas de saúde física, mas também o bem-estar psíquico, social e espiritual das pessoas, pacientes ou familiares. Tais iniciativas estão relacionadas à melhora no acesso, na cobertura e na qualidade da atenção à gestante e ao recém-nato (Brasil, 2004a).

2) Incentivo ao aleitamento materno: essa iniciativa se justifica por uma série de evidências científicas sobre os benefícios do aleitamento para o desenvolvimento infantil, os quais se relacionam ao fato de uma alimentação mais saudável para bebês até, no mínimo, seis meses, favorecer enormemente o vínculo afetivo entre o par mãebebê (Brasil, 2004a).

3) Acompanhamento do crescimento e desenvolvimento (Follow-up), estratégia que visa 
estreitar e manter o vínculo da criança e da família com os serviços de saúde, possibilitando ações de promoção da saúde, educação para hábitos de vida saudáveis, controle da vacinação, prevenção de problemas e de agravos ao desenvolvimento e a assistência necessária em tempo oportuno. Essa iniciativa permite às equipes de saúde acompanharem e registrarem o desenvolvimento infantil de zero à seis anos (Brasil, 2004b).

4) Atenção à saúde mental, que consiste no acompanhamento regular de crianças e suas famílias, permitindo a identificação de necessidades especiais pelas equipes de saúde mental, as quais devem estar capacitadas a intervir em situações que demandem uma abordagem mais específica, bem como a reconhecer sinais e sintomas indicativos de deficiências, psicopatologias e outras fontes de sofrimento psíquico (Brasil, 2004a).

5) Atenção à criança portadora de deficiência, que é o único tópico onde consta especificamente o termo “intervenção precoce”. Nesse ponto a cartilha traz um dado extremamente importante: segundo a Unicef, cerca de 70 a $80 \%$ das sequelas oriundas de deficiências poderiam ser evitadas ou minimizadas através de medidas preventivas e abordagem oportuna das alterações observadas (Brasil, 2004a).

6) Programa mãe-canguru, pois o método MãeCanguru, embora não conste na referida Agenda, foi instaurado pelo Ministério da Saúde como Norma de Atenção Humanizada ao RecémNascido de Baixo Peso no ano de 2000, e se configura como um programa de grande importância em algumas maternidades de referência para gestações de alto risco. Entre as vantagens desse método destacamos o estímulo ao vínculo mãe-bebê e ao aleitamento materno, um melhor controle térmico do bebê através do contato pele a pele com a mãe, a diminuição da incidência de infecções hospitalares e do tempo de permanência das internações, além do estímulo à maior competência e confiança dos pais no manuseio do seu filho de baixo peso, mesmo após a alta hospitalar (Brasil, 2002).

7) Estratégia de acolhimento mãe-bebê na unidade básica após a alta da maternidade, a qual tem como principal objetivo o estabelecimento do vínculo precoce da família com a unidade básica de saúde (UBS) mais próxima de sua residência, quando ao sair da maternidade a mãe recebe um cartão de referência da UBS que irá fazer o acompanhamento de sua saúde e de seu bebê. O acolhimento se caracteriza por ações como checagem e aplicação de vacinas, avaliação do aleitamento materno e do risco mãe-bebê, teste do pezinho e retirada dos pontos de cesariana, atendimentos de orientação para contracepção e vigilância nutricional, além do acompanhamento do crescimento e desenvolvimento do bebê por, no mínimo, três anos (Vilela, 2009).

Todas essas linhas de cuidado descritas acima dizem respeito a uma nova postura do sistema de saúde pública brasileiro no tocante às suas obrigações como prestador de serviços de saúde em conformidade com os direitos do cidadão previstos na Constituição. É disto que trata a Política Nacional de Humanização da Atenção e da Gestão do SUS-PNH (Brasil, 2004b), uma política que visa à valorização de todos os sujeitos envolvidos no processo de produção da saúde, que se dá em diferentes esferas, através de usuários, trabalhadores e gestores.

A PNH afirma a importância da dimensão subjetiva nas práticas de saúde, valorizando, assim, a dimensão humana no processo de produção de saúde. A compreensão desse processo é fundamental para uma mudança no paradigma assistencial em saúde, pois permite o reconhecimento de que a saúde, em sua dimensão mais ampla, não depende apenas da cura de patologias estabelecidas, mas principalmente de medidas de promoção e de proteção capazes de prevenir e minimizar a necessidade de medidas de reabilitação.

Sobretudo nesse aspecto, todas essas políticas e programas reunidos sob a égide do SUS se articulam diretamente com o campo das intervenções precoces, tema principal deste estudo. Embora não exista uma política específica de intervenção precoce que reúna em si ações voltadas especificamente para a prática nesse campo, verifica-se uma nítida ênfase em medidas preventivas, de valorização dos sujeitos e de compreensão de que saúde e doença envolvem complexos processos de produção intimamente ligados à dimensão subjetiva, além de uma crescente preocupação com reformas na área da saúde mental e da saúde materno-infantil, as quais se configuram como práticas de intervenção precoce.

Além disso, de um modo mais amplo, todo o movimento pela humanização do sistema de saúde constitui um campo fértil e contribui significativamente para a realização de práticas que se enquadram perfeitamente em medidas que se podem definir como intervenções precoces, ou seja, práticas pontuais com enfoque preventivo e planejadas a partir da singularidade de cada caso, porém baseadas numa 
abordagem mais ampla do sujeito, que leva em consideração suas dimensões biopsicossociais.

Com base nessa perspectiva e em levantamento bibliográfico sobre o tema, este artigo tem como foco discutir o tema das intervenções precoces em interface com a atuação profissional de psicólogos em saúde materno-infantil a fim de verificar as práticas de intervenção precoce em instituições públicas de saúde no Brasil, mais especificamente maternidades públicas de referência na cidade do Rio de Janeiro.

Considerando-se a importância da Psicologia como campo teórico e metodológico que pode colaborar no estudo e intervenção sobre o desenvolvimento humano e, sobretudo, sobre o campo da Psicologia Pediátrica, cujo objetivo é o estudo e a intervenção com enfoque interdisciplinar dedicada à atenção integral à saúde e ao desenvolvimento físico, cognitivo, social e emocional de gestantes, bebês, crianças, adolescentes e suas famílias (Crepaldi, Linhares \& Perosa, 2006), a principal questão que se coloca neste artigo é: “Como os psicólogos reconhecem e avaliam as práticas de intervenção precoce na sua atuação profissional, sobretudo quando trabalham na área da Saúde Materno-infantil, do prénatal ao follow up até os três anos de idade da criança?".

\section{MÉTODO}

Devidamente aprovada por Comitê de Ética (CAEE n. 0033.0.361.361-09), a pesquisa teve como base um delineamento descritivo-exploratório, com caráter qualiquantitativo, em que foram visitadas seis maternidades públicas de referência para gestação de risco na cidade do Rio de Janeiro. Inicialmente, por email ou por telefone, foram contatadas dez maternidades integrantes da Estratégia de Acolhimento Mãe-bebê da Secretaria Municipal de Saúde (SMS) do município do Rio de Janeiro (Vilela, 2009). Cabe esclarecer que fazem parte desse programa, ao todo, vinte e duas maternidades; no entanto, optou-se por selecionar unidades distribuídas por diferentes áreas programáticas (APs) com o intuito de obter-se uma amostra que representasse a realidade desse tipo de atendimento em saúde materno-infantil no município.

Dentro deste princípio, foram escolhidas duas unidades da AP 1.0 (nos bairros São Cristóvão e Saúde); duas unidades da AP 2.1 (nos bairros Flamengo e Laranjeiras); uma unidade da AP 2.2 (no bairro Vila Isabel); e uma unidade da AP 3.2 (no bairro Lins de Vasconcelos). Essas unidades representaram as regiões centro, zona sul e zona norte da cidade. Dessas unidades constavam dois hospitais universitários, um hospital geral, uma unidade técnicocientífica no âmbito da saúde materno-infantil e duas maternidades. Todas as unidades são consideradas como referência para gestações e partos de alto risco.

Adotou-se como critério de inclusão na amostra, para as maternidades, a instituição ser referência para atendimento à gestação e partos de alto risco, e para os psicólogos, trabalharem na instituição há mais de dois anos.

Para a coleta de dados foram utilizados um roteiro de entrevista semiestruturada e um protocolo de observação das maternidades, ambos elaborados pelas autoras. O roteiro de entrevista consistia de oito questões relativas ao conceito de Intervenções Precoces e suas possíveis articulações com a prática clínica dos psicólogos que trabalhavam nas maternidades públicas do Rio de Janeiro. O protocolo de observação foi construído de maneira a ser possível analisar a estrutura de serviços existentes e a capacitação da equipe técnica de cada instituição.

A coleta de dados foi realizada em visitas previamente agendadas, quando foram apresentados os objetivos da pesquisa e solicitadas autorização para realizar a coleta de dados na instituição e a assinatura do Termo de Consentimento Livre e Esclarecido. Nas visitas às maternidades foi aplicado o protocolo de observação e realizadas as entrevistas com os profissionais de Psicologia. Nas unidades em que funcionavam outros serviços além da maternidade, como os hospitais gerais, foram selecionados apenas os psicólogos responsáveis pelo atendimento no setor materno-infantil.

Os dados coletados foram processados diferentemente para cada tido de instrumento utilizado, e as entrevistas com os profissionais de Psicologia foram gravadas em áudio e posteriormente transcritas, a fim de se proceder à análise do relato verbal obtido, de acordo com a Metodologia de Análise de Conteúdo de Bardin (1977). Assim, o relato verbal dos participantes foi transcrito e analisado em sua totalidade, a fim de obter categorias significativas para a descrição objetiva, sistemática e quantitativa do conteúdo manifesto no relato verbal dos entrevistados, com posterior quantificação em subcategorias que permitissem obter informações mais precisas e objetivas sobre a frequência da ocorrência das características do conteúdo.

Dessa forma, chegou-se às seguintes categorias, com suas subcategorias e unidades de análise: 1 Identificação Geral: 1.1 - faixa etária: a) 31 a 35 anos; b) de 36 a 40 anos; c) acima de 40 anos; 1.2 - tempo de ingresso na instituição: a) 2 a 5 anos; 5 a 8 anos; c) 
acima de 8 anos; 1.3 - formação: a) superior completo; b) superior completo com especialização lato sensu; c) superior completo stricto sensu (mestrado); e 1.4 - experiência anterior: a) sim; b) não ; 2 - Conceito de Intervenção Precoce (IP): 2.1 definição de intervenção precoce: a) intervir no par mãe-bebê; b) detecção de riscos; c) acolhimento/escuta da mãe; d) reconhecer o bebê como "sujeito"; e) intervir sobre desvios de desenvolvimento; 3 - Prática Institucional de Intervenção Precoce: 3.1 - enfoque das práticas de IP: a) prática multiprofissional; b) prática profissional independente; 3.2 - práticas de IP identificadas pelos psicólogos: a) o próprio trabalho da Psicologia; b) enfoque da equipe voltado para o desenvolvimento precoce, 3.3 - avaliação dos resultados das práticas de IP adotadas: a) positiva; b) negativa; c) indiferente; e 3.4 - orientação institucional com ênfase em IP: a) não houve; b) investimentos da instituição na formação teórico-técnica (cursos); c) investimento da instituição em trabalho multiprofissional (troca com outros profissionais); 4 - Prática profissional do psicólogo e a relação com a Intervenção Precoce: 4.1 - abordagem teórico-conceitual em IP: a) Psicanálise; 4.2 formação teórico-metodológica que ampara a prática em IP: a) não teve formação suficiente na graduação; b) formação adquirida no percurso/experiência profissional; c) formação oferecida pela instituição (cursos de capacitação); d) formação acadêmica adquirida pelo psicólogo (cursos de especialização, residência, mestrado), 4.3 - profissionais que atuam em parceria com a Psicologia: a) enfermeiros; b) médicos; c) assistentes sociais; d) fonoaudiólogos; e) musicoterapeuta; e 4.4 - análise da relação entre IP e a Psicologia: a) todo trabalho do psicólogo é uma prática de IP; b) trabalho de acolhimento e escuta que o psicólogo realiza; e c) construção de um vinculo transferencial entre o psicólogo e o paciente.

No protocolo de observação da instituição foram identificados e analisados os quatro itens gerais relacionados a seguir, cujos dados foram registrados por frequência de ocorrência: 1 - Condições de conservação e limpeza das instituições visitadas, em que foram verificadas as características da estrutura física (edificações) das unidades, tais como a antiguidade do prédio, a presença de sujeira e detritos nos corredores e salas, infiltrações, etc.; 2 - Serviços prestados, em que se verificou a existência de determinados tipos de atendimentos, como pré-natal, grupos de gestantes, mãe-canguru, follow-up, ações educativas e outros; 3 - Atribuições do serviço de psicologia, em que foram levantadas as atuações do psicólogo em cada instituição, tais como a realização de atendimentos no pré-natal, consultas de seguimento, atendimentos em família, encaminhamentos para adoção, dentre outros; e 4 Número de psicólogos em cada instituição, em que se verificou quantos profissionais de Psicologia estavam alocados em cada maternidade e nos setores de atendimento materno-infantil dos hospitais gerais.

\section{RESULTADOS E DISCUSSÕES}

De acordo com as categorias descritas anteriormente, no que se refere aos dados coletados pelas entrevistas destaca-se que, do total de dez psicólogos entrevistados, quatro possuíam mais de quarenta anos de idade; seis trabalhavam na instituição durante períodos que variavam entre dois e cinco anos; oito possuíam nível superior completo com pósgraduação lato sensu (especializações em Psicologia Médica e Saúde Mental, por exemplo); três possuíam experiência anterior na área da saúde mental, dois na saúde materno-infantil e dois na área de Psicologia Médica e Hospitalar.

Quando questionados sobre o conceito de intervenção precoce, quatro profissionais a definiram como um trabalho focado no vínculo mãe-bebê, que tem a possibilidade de reorganizar situações que ameacem a saúde materno-infantil. Além disso, três entrevistados afirmaram que a intervenção precoce diz respeito ao trabalho com a mãe, através do acolhimento e da escuta; enquanto dois profissionais destacaram a importância do reconhecimento do bebê como sujeito e um relatou que a intervenção precoce se refere a um trabalho específico sobre os desvios do desenvolvimento.

Em relação à prática institucional de intervenções precoces, sete profissionais consideraram tratar-se de um trabalho com enfoque multiprofissional, embora quatro tenham afirmado que tais práticas estão subjacentes ao trabalho de todo profissional que se sensibilize para as questões do desenvolvimento infantil precoce; e quatro entrevistados relacionaram a prática de intervenção precoce mais especificamente ao trabalho dos profissionais de Psicologia da Maternidade. Do total de dez entrevistados, oito profissionais avaliaram as práticas de intervenção precoce adotadas na instituição como positivas, ou seja, como ações que visam minimizar as condições de riscos e, consequentemente, promover o desenvolvimento saudável. Três psicólogos entrevistados relataram não ter tido nenhum tipo de orientação por parte da instituição para trabalhar especificamente com o tema da intervenção precoce, 
embora outros três tenham mencionado que as instituições promoveram cursos diversos na área com temas que tangenciavam o trabalho em intervenção precoce, como, por exemplo, cursos sobre aleitamento materno e sobre humanização do parto, contribuindo, dessa maneira, para uma orientação institucional no campo da intervenção precoce.

Já no tocante ao item Prática profissional do psicólogo e a relação com a intervenção precoce, sete entrevistados consideraram seu próprio percurso profissional como a maior fonte de conhecimentos, teórico e prático, acerca desse tema, e todos adotavam o referencial teórico da Psicanálise para sustentar seu trabalho prático no campo da intervenção precoce. Igualmente, todos os psicólogos entrevistados $(n=10)$ concordaram que a graduação em Psicologia ofereceu pouco ou nenhum conhecimento sobre o campo, tanto no que se refere à abordagem teórico-conceitual quanto no que tange a uma formação teóricometodológica que amparasse a prática psicológica em intervenção precoce. Como área de interface multiprofissional que estabelece parceria com os psicólogos no campo da intervenção precoce, os entrevistados citaram o Serviço Social e a Enfermagem, cujos profissionais seriam os principais parceiros deles nesse tipo de prática, citados por três entrevistados cada; pela ordem do número de citações, em seguida foi mencionada a área médica, especificamente a Pediatria, (dois entrevistados) e as da Fonoaudiologia e da musicoterapia, (uma vez cada).

No que se refere à análise da relação entre o campo da intervenção precoce e o da Psicologia, seis entrevistados consideraram que a maior relação entre o trabalho do psicólogo e a pratica da intervenção precoce se refere às demandas especificas da atuação profissional do psicólogo, ou seja, além do trabalho de acolhimento e escuta que os profissionais de Psicologia realizam com os pacientes, a construção de um vínculo transferencial entre os profissionais de Psicologia e os pacientes é considerada como própria do trabalho de intervenção psicológica na área.

Tomando-se como base Bronfenbrenner (1979/1996), isto significa privilegiar o papel fundamental que as relações interpessoais desempenham na promoção do desenvolvimento. Para esse autor, são de extrema importância as relações entre as pessoas e a formação de díades com base em uma relação interpessoal de reciprocidade, como a díade mãe-bebê. Assim, a intervenção precoce realizada pelo psicólogo em instituições de saúde materno-infantil pode ser indicada como uma prática que favorece o desenvolvimento humano de uma maneira contextual, já que ocorre em um dos primeiros contextos ecológico-ambientais em que a díade mãe-bebê se insere quando do nascimento: a maternidade.

No que se refere aos dados coletados pelo protocolo de observação, observou-se que, das seis instituições visitadas, duas apresentavam excelentes condições de conservação e limpeza de suas instalações, já que suas edificações eram novas e/ou reformadas, não apresentando sujeira aparente nos corredores, salas, banheiros, etc.; e outras duas apresentaram boas condições, e se encontravam, no momento, em obras para melhorias.

Todas as instituições ofereciam atendimento prénatal, parto e acompanhamento posterior (follow-up), e apenas uma não possuía grupo de gestantes; ademais, todas eram consideradas instituições de referência para gestação de alto risco. Os serviços de Psicologia dessas instituições atendiam a todas as pacientes desde o pré-natal até alguns meses após o parto; no entanto, em duas instituições o setor de Psicologia não possuía uma sala própria para atendimento. No tocante ao número de profissionais de Psicologia, o hospital geral contava com o maior número de psicólogos $(\mathrm{n}=19)$ trabalhando no setor materno-infantil, seguindo-se os hospitais universitários, com sete psicólogos em cada um, e uma das maternidades visitadas, que possuía apenas um profissional de psicologia.

O trabalho dos profissionais de Psicologia nas maternidades públicas do Rio de Janeiro tem sido cada vez mais requisitado, inclusive, por causa da atual preocupação do Sistema Único de Saúde Brasileiro em humanizar os atendimentos à saúde da população, de acordo com os princípios da Política Nacional de Humanização da Atenção e da Gestão do SUS - PNH (Brasil, 2004b).

Nessa perspectiva, a intervenção precoce estaria de acordo com os princípios fundamentais da $\mathrm{PNH}$, quando se propõe não só minimizar potenciais riscos, mas também produzir mecanismos que garantam maior e melhor qualidade de saúde aos indivíduos, focalizando a prevenção aos fatores de risco para problemas de desenvolvimento infantil, como indicam Graminha e Martins (1997).

Dessa forma, os dados aqui apresentados refletem certa concordância entre as concepções sobre intervenção precoce dos profissionais de Psicologia entrevistados e a própria definição conceitual dessa prática, já que, segundo Franco (2007), a intervenção precoce se define como uma possibilidade intencional de intervir junto ao desenvolvimento infantil, de 
maneira a garantir que este processo ocorra da melhor maneira possível.

Nesse sentido, o trabalho da Psicologia em instituições de saúde materno-infantil toma lugar de relevância, já que o psicólogo detém (ou deveria deter) um conhecimento teórico-prático em intervenção precoce que certamente responde ao objetivo de prevenção dessa prática e, por vezes, figura na instituição sem ser denominada como tal. Isto corrobora a hipótese de que tais práticas deveriam ser legitimadas e mais incentivadas nas maternidades públicas do Rio de Janeiro, como destacam Graminha e Martins, (1997) e Delvan, Menezes, Geraldi e Albuquerque (2009).

A maioria dos profissionais considerou que a articulação entre a Psicologia e intervenção precoce ocorre, em grande parte, devido às demandas próprias da profissão, tais como a facilitação do laço transferencial entre o psicólogo e seus pacientes e o trabalho de escuta clínica e acolhimento realizado por estes profissionais junto aos pacientes. Neste sentido, esses profissionais avaliaram que a prática de intervenção precoce é inerente ao exercício profissional do psicólogo em saúde materno-infantil. Para autores como Delvan et al. (2009), é de grande relevância a presença desses profissionais neste segmento da saúde pública, já que a atuação de psicólogos em Pediatria é capaz de promover intervenções eficazes e acessíveis tanto a crianças e adolescentes quanto aos seus familiares.

O fato de todos os entrevistados concordarem que os cursos de graduação oferecem pouca ou nenhuma informação sobre este campo de atuação é um dado alarmante, indicando a necessidade urgente de as instituições de ensino superior prestarem atenção à carência de conteúdos curriculares específicos para a formação nessa área, uma vez que há um número crescente de profissionais de Psicologia que ingressam em instituições de saúde pública, inclusive na área da saúde materno-infantil.

Do mesmo modo, as instituições de formação acadêmico-profissional deveriam considerar seriamente o risco de estar formando profissionais despreparados para atuar em um campo tão delicado com o é o da saúde materno-infantil. Tal situação torna-se ainda mais grave quando se constata que as instituições de saúde a que esses profissionais estão vinculados também não oferecem capacitação específica sobre o tema, conquanto muitos profissionais reconheçam uma aproximação possível quando são promovidos debates sobre temas como amamentação, método canguru e humanização, temas tão importantes para o desenvolvimento infantil e abordados pela PNH (Brasil, 2004b).

Cabe destacar ainda, para além da formação profissional, a importância do treinamento em serviço e de cursos de atualização não só para psicólogos, mas também para os profissionais das equipes de saúde em geral. Particularmente em maternidades, isto serviria para despertar um olhar mais sensível aos sinais indicativos de risco ao desenvolvimento infantil, possibilitando, assim, encaminhamentos mais acolhedores das questões relativas aos momentos pré, peri e pós-natal, valorizando, dessa forma, a delicadeza própria desse período de desenvolvimento humano e os cuidados especiais de que necessitam tanto o bebê como sua mãe.

\section{REFERÊNCIAS}

Bardin L. (1977). Análise de Conteúdo. Lisboa, Portugal: Edições 70.

Brasil. Ministério da Saúde. (2002). Atenção Humanizada ao RecémNascido de Baixo Peso: Método Mãe Canguru: Manual de Curso. Brasília: Ministério da Saúde, Secretaria de Atenção à Saúde. Área Técnica da Saúde. Recuperado em 03 de maio, 2010, de http://www.fiocruz.br/redeblh/media/manualcanguru.pdf;

Brasil. Ministério da Saúde. (2004a). Agenda de compromissos para a saúde integral da criança e redução da mortalidade infantil. Brasília: Ministério da Saúde, Secretaria de Atenção à Saúde, Departamento de Ações Programáticas Estratégicas. Recuperado em 13 de julho, 2010, de http://bvsms.saude.gov.br/bvs/ publicacoes/agenda_compro_crianca.pdf;

Brasil. Ministério da Saúde. (2004b). HumanizaSUS: Política Nacional de Humanização: a humanização como eixo norteador das práticas de atenção e gestão em todas as instâncias do SUS. Brasília: Ministério da Saúde, Secretaria-Executiva, Núcleo Técnico da Política Nacional de Humanização. Recuperado em 12 de julho, 2010, de http://bvsms.saude.gov.br/bvs/publicacoes/ humanizasus_2004.pdf;

Bronfenbrenner, U. (1979/1996). A Ecologia do Desenvolvimento Humano: Experimentos Naturais e Planejados. Porto Alegre, Artes Médicas;

Bronfenbrenner, U. (1989). Ecological systems theory. Annals of Child Development, Greenwich, CT, JAI Press, 6, p. 187-249;

Crepaldi, M. A.; Linhares, M. B. M. \& Perosa, G. B. (Orgs.) (2006). Temas em Psicologia Pediátrica. São Paulo: Casa do Psicólogo;

Delvan, J. S; Menezes, M; Geraldi P. A. \& Albuquerque, L. B. G. (2009, setembro/dezembro). Estimulação precoce com bebês e pequenas crianças hospitalizadas: uma intervenção em psicologia pediátrica. Contrapontos, 9 (3). Recuperado em 16 de dezembro, 2010, de https://www6.univali.br/seer/index.php/rc/article/ view/1453/1473;

Franco, V. (2007). Dimensões transdisciplinares do trabalho de equipe em intervenção precoce. Interação em Psicologia, 11. Recuperado em 22 de abril, 2010, de http://ojs.c3sl.ufpr.br/ojs2/index.php/psicologia/article/view/6452/ 6779; 
Graminha, S. S. V \& Martins, M. A. O. (1977). Condições adversas na vida de crianças com atraso no desenvolvimento. Medicina, 30, 259-267;

Hallal, C. Z; Marques, N. R \& Braccialli, L. M. P. (2008). Aquisição de habilidades funcionais na área de mobilidade em crianças atendidas em um programa de estimulação precoce. Revista Brasileira de Crescimento e Desenvolvimento Humano, 18 (1). Recuperado em 22 de abril, 2010, de http://pepsic.bvsalud.org/pdf/rbcdh/v18n1/05.pdf;

Lampreia, C. (2007). A perspectiva desenvolvimentista para a intervenção precoce no autismo. In: Estudos de Psicologia, Campinas: v. 24(1): [online] Jan- mar/2007. [acesso em 28 abr. 2010] pp. 105-114. Disponível em: http://www.scielo.br/pdf/estpsi/v24n1/v24n1a12.pdf;

Martins, E. \& Szymanski, H. (2004). A abordagem ecológica de Urie Bronfenbrenner em estudos com famílias. Estudos e Pesquisas em Psicologia, 4(1). Recuperado 26 de outubro, 2010, de http://pepsic.bvsalud.org/scielo.php?script=sci_arttext\&pid=S180 8-42812004000100006;
Tegethof, M. I. S. C. A. (2007). Estudos sobre a intervenção precoce em Portugal: ideias dos especialistas, dos profissionais e das famílias. Tese de Doutorado. Faculdade de Psicologia e de Ciências da Educação, Universidade do Porto, Portugal. Recuperado em 01 de maio, 2010, de http://repositorio.ispa.pt/bitstream/10400.12/47/1/TES\%20TEGE 1\%20-\%2017453.pdf;

Vilela, M. A. (2009). As ações direcionadas a saúde da criança na secretaria municipal de saúde. In M. A. Vilela. Capacitação em Saúde Mental para Intervenções Precoces, (pp. 20-32). Rio de Janeiro: ABENEPI;

\section{Endereço para correspondência:}

Ana Cristina Barros da Cunha. Depto de Psicologia Clínica, Universidade Federal do Rio de Janeiro - UFRJ. Pavilhão Nilton Campos - Campus Praia Vermelha. Av. Pasteur, 250, Urca, CEP 22290-240, Rio de Janeiro-RJ, Brasil.E-mail: acbcunha@yahoo.com.br. 